Managing Cancer

Breakthrough Pain 


\section{Managing Cancer Breakthrough Pain}

Donald R Taylor, MD

Comprehensive Pain Care, PC

Taylor Research, LLC

Marietta, GA

Springer Healthcare 
Published by Springer Healthcare Ltd, 236 Gray's Inn Road, London, WC1X 8HB, UK.

www.springerhealthcare.com

(c) 2013 Springer Healthcare, a part of Springer Science+Business Media.

All rights reserved. No part of this publication may be reproduced, stored in a retrieval system or transmitted in any form or by any means electronic, mechanical, photocopying, recording or otherwise without the prior written permission of the copyright holder.

British Library Cataloguing-in-Publication Data.

A catalogue record for this book is available from the British Library.

ISBN 978-1-908517-76-0

Although every effort has been made to ensure that drug doses and other information are presented accurately in this publication, the ultimate responsibility rests with the prescribing physician. Neither the publisher nor the authors can be held responsible for errors or for any consequences arising from the use of the information contained herein. Any product mentioned in this publication should be used in accordance with the prescribing information prepared by the manufacturers. No claims or endorsements are made for any drug or compound at present under clinical investigation.

Project editor: Tess Salazar

Designer: Joe Harvey

Artworker: Sissan Mollerfors

Production: Marina Maher

Printed in Great Britain by Latimer Trend \& Company Ltd. 


\section{Contents}

Author biography vii

Abbreviations viii

Glossary ix

Preface xiii

1 Introduction 1

Experiencing the multidimensional aspects of pain as a patient with cancer 1

Historical perspective $\quad 2$

Pain flares and their treatment $\quad 8$

References

2 What is cancer breakthrough pain? 13

Definitions 13

$\begin{array}{ll}\text { Characteristics of cancer breakthrough pain } & 17\end{array}$

$\begin{array}{ll}\text { Assessment of cancer breakthrough pain } & 18\end{array}$

$\begin{array}{ll}\text { Understanding cancer breakthrough pain and its treatment } & 19\end{array}$

$\begin{array}{ll}\text { References } & 25\end{array}$

3 Medications for cancer breakthrough pain 29

$\begin{array}{ll}\text { Introduction to rapid-onset opioids } & 29\end{array}$

Rapid-onset opioids $\quad 40$

$\begin{array}{ll}\text { Summary } & 60\end{array}$

$\begin{array}{ll}\text { References } & 60\end{array}$

4 Practical approach to the management of $\begin{array}{ll}\text { cancer breakthrough pain } & 67\end{array}$

Practical approach to the management of cancer breakthrough pain $\quad 67$

$\begin{array}{ll}\text { Case studies } & 70\end{array}$

$\begin{array}{lr}\text { References } & 80\end{array}$ 
$\mathrm{VI} \cdot$ CONTENTS

5 Abuse, aberrant drug behavior, diversion, and addiction 83 Definitions

Patients at risk 83

Screening prior to initiating a rapid-onset opioid 86

References

6 TIRF REMS Access program

What are REMS and TIRF REMS?

What are the goals of the TIRF REMS Access program?

Efforts to enhance patient safety

TIRF REMS Access program and accessibility to treatment 92

References

7 Summary

Appendix 95

References

98 


\section{Author biography}

Donald Taylor, MD, is the Medical Director at Comprehensive Pain Care, PC, and the Principal Investigator for Taylor Research, LLC. He graduated from the Medical College of Georgia in Augusta, GA in 1984. He then completed a residency in Anesthesiology and Critical Care Medicine at Johns Hopkins Hospital in Baltimore, MD. After this, he stayed at Johns Hopkins Hospital for a fellowship in Regional Anesthesia and Pain Management. During his fellowship he established the use of patient-controlled analgesia and epidural analgesia for postoperative pain at Johns Hopkins Hospital. After his fellowship was completed, he remained on the faculty for a year in the division of Regional Anesthesia and Pain Management, providing services in the operating room and in the pain clinic. In 1990, he left Johns Hopkins Hospital and returned to his native Georgia where he entered the private practice of Anesthesiology and Pain Medicine. He has spent the past 22 years practicing pain medicine and conducting Phase II and III clinical trials for pain management medications and devices. His interest in the treatment of cancer-related pain began in his residency when he saw the great relief of suffering that regional anesthetic techniques and skillfully administered medications could bring to patients with cancer. He continues to care for such patients today and has been involved in Phase III clinical trials of several of the newest class of medications, known today as the rapid-onset opioids, developed specifically for the management of cancer breakthrough pain. 


\section{Abbreviations}

ATC

BGPF

BTP

cBTP

FDA

FBSF

FBT

FPNS

FSS

FST

INFS

LAO

NRS

ORT

OTFC

REMS

ROO

SAO

SOAPP-R

TENS

TIRF

WHO around-the-clock

background pain flare

breakthrough pain

cancer breakthrough pain

US Food and Drug Administration

fentanyl buccal soluble film

fentanyl buccal tablet

fentanyl pectin nasal spray

fentanyl sublingual spray

fentanyl sublingual tablet

intranasal fentanyl spray

long-acting opioids

numerical rating scale

Opioid Risk Tool

oral transmucosal fentanyl citrate

Risk Evaluation and Mitigation Strategies

rapid-onset opioids

short-acting opioids

Screener and Opioid Assessment for Patients

with Pain-Revised

transcutaneous electrical nerve stimulation

Transmucosal Immediate Release Fentanyl

World Health Organization 


\section{Glossary}

Aberrant drug behavior Any medication-related behaviors that depart from strict adherence to the prescribed therapeutic plan of care.

Abuse The use of an illicit drug or the intentional self-administration of a prescription (or over-the-counter) medication for any nonmedical purpose, such as altering one's state of consciousness (eg, "getting high").

Addiction A primary, chronic disease of brain reward, motivation, memory, and related circuitry. Dysfunction in these circuits leads to characteristic biological, psychological, social, and spiritual manifestations.

Adequate pain control Pain is adequately controlled when it is under "good enough" control to allow the patient to engage in reasonable desired goals. For many patients this will correspond to a pain score on the 0-10 Numerical Rating Scale (NRS) of 0-4.

Around-the-clock (ATC) Refers to a medication given on a time contingent basis over a 24 -hour period. The patient may need to be awakened to administer drug.

Background pain Pain that persist for at least 12 hours and usually lasts for 24 hours out of a day (also known as basal pain or persistent pain).

Background pain flare (BGPF) Flare of increased pain that occurs before the background pain is under adequate control. These flares of pain are treated with a rescue dose of regular-release opioid (a short-acting opioid [SAO]). Typically, the rescue medication is an additional dose of the same drug administered ATC for background pain control.

Basal pain See "Background pain".

Cancer pain Pain associated with cancer or its treatment. Pain associated with cancer can have multiple causes, such as disease progression, treatment (eg, radiation, chemotherapy, surgery), and concurrent diseases (eg, arthritis, lumbar disc disease). 
Cancer breakthrough pain (cBTP) A transient exacerbation of pain that occurs in the setting of generally adequately controlled background pain. cBTP is typically of rapid onset and severe in intensity, and generally self-limiting with an average duration of 30 minutes. cBTP episodes can occur spontaneously or in relation to a specific predictable (eg, walking) or unpredictable (eg, coughing) trigger or incident.

Diversion The intentional removal of a medication from legitimate distribution and dispensing channels. Diversion also involves the sharing or purchasing of prescription medication between family members and friends, or individual theft from family and friends.

Efficacy The ability of a drug to produce the desired therapeutic effect.

End-of-dose failure Occurs when the serum level of an analgesic falls below the therapeutic level before the next timed dose; pain emerges and mimics cBTP.

Equianalgesic A dose of one analgesic that is equivalent in pain-relieving effects to that of another analgesic. This equivalence allows for substitution of one analgesic for another at an equally effective dose.

Intermittent pain Intermittent flares of pain in patients with cancer who do not have background pain. This pain is very similar in characteristics to $\mathrm{CBTP}$, but as there is no background pain, this pain cannot be cBTP or BGPF. Also known as "nonbreakthrough pain".

Long-acting opioid (LAO) Pharmacologically long-acting opioid or an opioid with a modified delivery system designed to provide longlasting (8-24 hours) relief from a single dose.

Opioid agreement An agreement that defines patient responsibilities when receiving chronic opioid treatment; responsibilities include, for example, the patient's agreement to submit to urine drug testing and random pill counts.

Opioid consent form An informed consent form for a patient who is being prescribed chronic opioids.

Opioid-risk tool (ORT) A written risk-assessment questionnaire (5 questions) designed to assess the risk for aberrant drug behavior prior to prescribing chronic opioid treatment. 
Opioid tolerant Patients are considered opioid tolerant if they have been taking at least $60 \mathrm{mg}$ of oral morphine (or an equivalent dose of another opioid) daily for at least 1 week.

Persistent pain See "Background pain".

Potency The relationship between the dose of a drug and the therapeutic effect (ie, the drug's strength). A drug is considered potent when a small amount of the drug achieves the intended effect.

Predictable cBTP cBTP that is incident-related and occurs consistently with an activity.

Risk Evaluation and Mitigation Strategy (REMS) A risk management plan that uses risk-minimization strategies beyond approved labeling to manage serious risks associated with a drug. The US Food and Drug Administration (FDA) requires that all physicians, pharmacists, and patients prescribing, dispensing, and using ROOs (respectively) are registered on REMS.

Rescue dose/medication Short-acting opioids (SAO) used to treat BGPFs and used as part of the titration protocol for the ATC analgesic. Typically, the rescue dose is $5-20 \%$ of the total ATC dose.

Rapid-onset opioid (ROO) A class of drugs with onset of analgesic action in less than 20 minutes. Currently, all FDA-approved drugs in this class consist of fentanyl and are administered via a variety of transmucosal delivery systems.

Short-acting opioids (SAO) Regular-release opioids that are not pharmacologically long-acting.

Screener and Opioid Assessment for Patients with Pain-Revised (SOAPP-R) A written screening form that predicts the risk of opioid abuse in patients with chronic pain.

Spontaneous cBTP cBTP that occurs without any inciting incident; it is unpredictable.

\section{Transmucosal Immediate Release Fentanyl Risk Evaluation} and Mitigation Strategy (TIRF REMS) FDA-mandated educational program for physicians, pharmacists, and patients aimed at mitigating the risks of misuse, abuse, addiction, overdose, and serious complications due to medication errors associated with ROOs. 
World Health Organization (WHO) pain ladder Recommendations for cancer pain management by the WHO in a 3-step ladder format:

1. First step for mild pain: nonsteroidal anti-inflammatory drugs or acetaminophen.

2. Second step for moderate pain: add a "weak opioid" (less potent $\mu$-agonist).

3. Third step for severe pain: change opioid to a "strong opioid" (ie, a potent full $\mu$-agonist).

Urine drug testing A test to screen for the presence of administered drugs and the absence of nonprescribed or illegal drugs. Other body fluids can be used as well (eg, saliva or blood). 


\section{Preface}

Cancer pain management is a broad and vastly rich topic. This book focuses on one narrow, but important, aspect of cancer pain management: cancer breakthrough pain (cBTP). It is assumed that the reader has a passing familiarity with acute and chronic cancer pain and its management, but would like to have a better understanding of cBTP and the new class of drugs - the rapid-onset opioids (ROOs) - developed specifically for the management of cBTP.

The purpose of this book is to provide healthcare professionals with clinically relevant information for understanding and managing cBTP. Early chapters will discuss the history of cBTP treatment and will explain a practical definition of $\mathrm{CBTP}$ suitable for caring for the patient with cancer. Subsequent chapters will be devoted to reviewing assessment techniques and widely accepted treatment recommendations for cBTP. Particular emphasis will be placed on current state-of-the-art medications (ie, ROOs) approved for the management of cBTP. The risks associated with ROOs, including abuse, diversion, and addiction, will be explored as well. Lastly, the book will explain the federally mandated Risk Evaluation and Mitigation Strategies (REMS), and how REMS plays a role in attenuating some of the risks associated with ROOs.

The author hopes that this concise book will help fill some of the gaps in knowledge that may exist with regards to cBTP and its treatment. 